\title{
Solitons, Rogues and Interaction Behaviors of Third- Order Nonlinear Schrodinger Equation
}

Ren Bo ( $\square$ renbosemail@163.com )

Zhejiang University of Technology

Shi Kai-Zhong

Zhejiang University of Technology

Shou-Feng Shen

Zhejiang University of Technology

Wang Guo-Fang

Zhejiang University of Technology

Peng Jun-Da

Zhejiang University of Technology

Wang Wan-Li

Zhejiang University of Technology

\section{Research Article}

Keywords: Third-order NLSE, Hirota bilinear method, Long wave limit method, Soliton, Rogue wave

Posted Date: January 5th, 2022

DOI: https://doi.org/10.21203/rs.3.rs-1201398/v1

License: (c) (i) This work is licensed under a Creative Commons Attribution 4.0 International License.

Read Full License 


\section{Solitons, rogues and interaction behaviors of third-order nonlinear Schrödinger equation}

Kai-Zhong Shi, Bo Ren, ${ }^{\text {a) }}$ Shou-Feng Shen, Guo-Fang Wang, Jun-Da Peng, and Wan-Li Wang

Department of Mathematics, Zhejiang University of Technology, Hangzhou 310014, China

(Dated: 31 December 2021)

In this paper, we investigate the third-order nonlinear Schrödinger equation which is used to describe the propagation of ultrashort pulses in the subpicosecond or femtosecond regime. Based on the independent transformation, the bilinear form of the third-order NLSE is constructed. The multiple soliton solutions are constructed by solving the bilinear form. The multi-order rogue waves and interaction between one-soliton and first-order rogue wave are obtained by the long wave limit in multisolitons. The dynamics of the first-order rogue wave, second-order rogue wave and interaction between one-soliton and first-order rogue wave are presented by selecting the appropriate parameters. In particular parameters, the positions and the maximum of amplitude of rogue wave can be confirmed by the detail calculations.

PACS numbers: 02.30.Ik, 05.45.Yv.

Keywords: Third-order NLSE, Hirota bilinear method, Long wave limit method, Soliton, Rogue wave

a) Author to whom correspondence should be addressed; Electronic mail: renbosemaila163.3om 


\section{INTRODUCTION}

Recently, finding exact solutions of nonlinear partial differential equations plays an important part in nonlinear science and engineering ${ }^{1-4}$. Among these exact solutions, solitary and rogue waves have already gained widely applications in studying natural phenomena, such as fluids, engineering, nonlinear optics and plasmas ${ }^{5-8}$. The phenomena of rogue waves can create huge water walls taller than 20-30 meters and make great damage to ships, coastal oil platform and marine industries ${ }^{9-11}$. Sometimes these massive rogue waves will become tsunamis, due to underwater disturbance such as earthquakes, volcanic eruptions, typically with the aid of nuclear explosion or asteroids ${ }^{10}$. There are many powerful methods proposed for rogue wave solution, such as the Hirota bilinear method ${ }^{12-23}$, the Darboux transformation ${ }^{24,25}$ and the extended homoclinic test method ${ }^{26}$. To describe complex physical phenomena, the interactions between multi-solitons and rogue waves are getting more attention.

In nonlinear theory, the nonlinear Schrödinger equation (NLSE) is one of a traditional equation, has been widely studied in some literatures as a model of soliton transmission in the optical fiber ${ }^{21,27-29}$. The generalized NLSE has been a considerable interest in the experimental and theoretical of Bose-Einstein condensates ${ }^{30}$. The $(1+1)$-dimensional NLSE has the following form

$$
i u_{t}+u_{x x}+2|u|^{2} u=0
$$

The higher order nonlinear terms in Eq. (1) are increased with the transmission speed of optical pulses in optical fibres in the picosecond or femtosecond regime ${ }^{27,31}$. This phenomena have been observed in both experimental and numerical simulations.

In this paper, we consider a third-order nonlinear Schrödinger equation (NLSE) with the following form ${ }^{21,32}$,

$$
i u_{x}-\alpha_{2}\left(u_{t t}-2 u|u|^{2}\right)+i \alpha_{3}\left(u_{t t t}-6 u_{t}|u|^{2}\right)=0
$$

where $u(x, t)$ represents the soliton envelope amplitude, $\alpha_{2}$ is the group velocity dispersion and $\alpha_{3}$ is the third-order dispersion. Our main goal is to obtain the multi-solitons, multiorder rogue waves and their interaction solutions by using the Hirota bilinear method and long wave limit. 
With the aid of transformation

$$
u=\frac{g}{f}
$$

Eq. (2) is transformed into the following bilinear form

$$
\begin{aligned}
& \left(i \alpha_{3} D_{t}^{3}-\alpha_{2} D_{t}^{2}-i 6 \rho \rho^{*} \alpha_{3} D_{t}+i D_{x}\right) g \cdot f=-2 \rho \rho^{*} \alpha_{2} g f, \\
& D_{t}^{2} f \cdot f=2 \rho \rho^{*} f^{2}-2 g g^{*}
\end{aligned}
$$

where $D_{x}$ and $D_{t}$ are the Hirota bilinear operators defined by

$$
D_{x}^{n} D_{t}^{m} f \cdot g=\left.\left(\partial_{x}-\partial_{x^{\prime}}\right)^{n}\left(\partial_{t}-\partial_{t^{\prime}}\right)^{m} f(x, t) g\left(x^{\prime}, t^{\prime}\right)\right|_{x^{\prime}=x, t^{\prime}=t},
$$

and $\rho$ is an arbitrary constant and the asterisks denote complex conjugate.

This paper is organized as follows. In Sec. II, the multi-solitons of Eq. (4) are given by using the Hirota bilinear method. By taking $N=4$ and selecting the appropriate parameters, the dynamics of four-soliton solution is presented in Fig. 1. In Sec. III, the multi-order rogue waves of the third-order NLSE are obtained by using long wave limit to multi-soliton solutions. By taking $M=1, N=2$ and $M=2, N=4$ and selecting the appropriate parameters, first-order and second-order rogue wave solutions are given. In Sec. IV, the interaction between one-soliton and first-order rogue wave is presented by selecting $N=3$ in the multi-solitons. The last section contains a conclusion.

\section{MULTI-SOLITON SOLUTIONS OF THIRD-ORDER NLSE}

In this section, we shall construct the multi-soliton solutions of Eq. (2) by taking the Hirota bilinear method. The auxiliary functions $f$ and $g$ assume the expansions form in terms of $\epsilon$ by the Hirota bilinear method ${ }^{21}$

$$
\begin{aligned}
& f=1+\epsilon f_{1}+\epsilon^{2} f_{2}+\epsilon^{3} f_{3}+\cdots+\epsilon^{n} f_{n}+\cdots, \\
& g=g_{0}\left(1+\epsilon g_{1}+\epsilon^{2} g_{2}+\epsilon^{3} g_{3}+\cdots+\epsilon^{n} g_{n}+\cdots\right) .
\end{aligned}
$$

Substituting Eq. (6) into the bilinear form (4) and eliminating the coefficients of all powers of $\epsilon$, the series of equations are obtained. By solving these equations, the multi-soliton 
solutions are obtained

$$
\begin{aligned}
& f_{N}=\sum_{\mu=0,1} \exp \left(\sum_{i=1}^{N} \mu_{i} \xi_{i}+\sum_{i<j}^{(N)} \mu_{i} \mu_{j} B_{i j}\right), \\
& g_{N}=g_{0} \sum_{\mu=0,1} \exp \left(\sum_{i=1}^{N} \mu_{i}\left(\xi_{i}+A_{i}\right)+\sum_{i<j}^{(N)} \mu_{i} \mu_{j} B_{i j}\right),
\end{aligned}
$$

where

$$
\begin{aligned}
g_{0} & =\rho \exp \left(i 2 \rho \rho^{*} x\right), \\
\xi_{i} & =\omega_{i} t+k_{i} x+\phi_{i}, \\
e^{A_{i}} & =\frac{i 6 \omega_{i} \alpha_{3} \rho \rho^{*}-i \omega_{i}^{3} \alpha_{3}-\alpha_{2} \omega_{i}^{2}-i k_{i}}{i 6 \omega_{i} \alpha_{3} \rho \rho^{*}-i \omega_{i}^{3} \alpha_{3}+\alpha_{2} \omega_{i}^{2}-i k_{i}}, \\
e^{B_{i j}} & =\frac{18 \rho \rho^{*} \alpha_{3}^{2} \omega_{i}^{2} \omega_{j}^{2}\left(\omega_{i}-\omega_{j}\right)^{2}-N_{i j}-M_{i j}-\left(k_{i} \omega_{j}-k_{j} \omega_{i}\right)^{2}}{18 \rho \rho^{*} \alpha_{3}^{2} \omega_{i}^{2} \omega_{j}^{2}\left(\omega_{i}+\omega_{j}\right)^{2}-N_{i j}^{\prime}-M_{i j}^{\prime}-\left(k_{i} \omega_{j}-k_{j} \omega_{i}\right)^{2}}, \\
N_{i j} & =\omega_{i}^{2} \omega_{j}^{2}\left(\omega_{i}-\omega_{j}\right)^{2}\left[\alpha_{3}\left(\omega_{i}-\omega_{j}\right)^{2}+\omega_{i} \omega_{j}+\alpha_{2}^{2}\right] \\
N_{i j}^{\prime} & =\omega_{i}^{2} \omega_{j}^{2}\left(\omega_{i}+\omega_{j}\right)^{2}\left[\alpha_{3}\left(\omega_{i}+\omega_{j}\right)^{2}-\omega_{i} \omega_{j}+\alpha_{2}^{2}\right] \\
M_{i j} & =\alpha_{3} \omega_{i} \omega_{j}\left(\omega_{i}-\omega_{j}\right)\left[k_{j} \omega_{i}^{2}+2 \omega_{i} \omega_{j}\left(k_{i}-k_{j}\right)-k_{i} \omega_{j}^{2}\right], \\
M_{i j}^{\prime} & =\alpha_{3} \omega_{i} \omega_{j}\left(\omega_{i}+\omega_{j}\right)\left[k_{j} \omega_{i}^{2}+2 \omega_{i} \omega_{j}\left(k_{i}+k_{j}\right)+k_{i} \omega_{j}^{2}\right], \\
k_{i} & =\omega_{i}\left(6 \rho \rho^{*} \alpha_{3}-\alpha_{3} \omega_{i}^{2} \pm \sqrt{4 \rho \rho^{*} \alpha_{2}^{2}-\alpha_{2}^{2} \omega_{i}^{2}}\right),
\end{aligned}
$$

and $\omega_{i}, k_{i}$ and $\phi_{i}$ are arbitrary constants. The notation $\sum_{\mu=0,1}$ indicates summation over all possible combinations of $\mu_{i}=0,1,(i=1,2, \cdots, N)$. The summation $\sum_{i<j}^{(N)}$ is over all possible combinations of the $N$ elements with the specific condition $i<j$.

One take the four-soliton solution of Eq. (2) as an example by taking $N=4$ to Eq. (7). The four-soliton solution reads as

$$
\begin{array}{r}
f=1+f_{1}+f_{2}+f_{3}+f_{4}, \\
g=g_{0}\left(1+g_{1}+g_{2}+g_{3}+g_{4}\right),
\end{array}
$$


where

$$
\begin{aligned}
f_{1}= & e^{\xi_{1}}+e^{\xi_{2}}+e^{\xi_{3}}+e^{\xi_{4}}, \\
f_{2}= & e^{\xi_{1}+\xi_{2}+B_{12}}+e^{\xi_{1}+\xi_{3}+B_{13}}+e^{\xi_{1}+\xi_{4}+B_{14}} \\
& +e^{\xi_{2}+\xi_{3}+B_{23}}+e^{\xi_{2}+\xi_{4}+B_{24}}+e^{\xi_{3}+\xi_{4}+B_{34}}, \\
f_{3}= & e^{\xi_{1}+\xi_{2}+\xi_{3}+B_{12}+B_{13}+B_{23}}+e^{\xi_{1}+\xi_{2}+\xi_{4}+B_{12}+B_{14}+B_{24}} \\
& +e^{\xi_{1}+\xi_{3}+\xi_{4}+B_{13}+B_{14}+B_{34}}+e^{\xi_{2}+\xi_{3}+\xi_{4}+B_{23}+B_{24}+B_{34}}, \\
f_{4}= & \xi^{\xi_{1}+\xi_{2}+\xi_{3}+\xi_{4}+B_{12}+B_{13}+B_{14}+B_{23}+B_{24}+B_{34}}, \\
g_{1}= & e^{\xi_{1}+A_{1}}+e^{\xi_{2}+A_{2}}+e^{\xi_{3}+A_{3}}+e^{\xi_{4}+A_{4}}, \\
g_{2}= & e^{\xi_{1}+\xi_{2}+A_{1}+A_{2}+B_{12}}+e^{\xi_{1}+\xi_{3}+A_{1}+A_{3}+B_{13}}+e^{\xi_{1}+\xi_{4}+A_{1}+A_{4}+B_{14}} \\
& +e^{\xi_{2}+\xi_{3}+A_{2}+A_{3}+B_{23}}+e^{\xi_{2}+\xi_{4}+A_{2}+A_{4}+B_{24}}+e^{\xi_{3}+\xi_{4}+A_{3}+A_{4}+B_{34}}, \\
g_{3}= & e^{\xi_{1}+\xi_{2}+\xi_{3}+A_{1}+A_{2}+A_{3}+B_{12}+B_{13}+B_{23}}+e^{\xi_{1}+\xi_{2}+\xi_{4}+A_{1}+A_{2}+A_{4}+B_{12}+B_{14}+B_{24}} \\
& +e^{\xi_{1}+\xi_{3}+\xi_{4}+A_{1}+A_{3}+A_{4}+B_{13}+B_{14}+B_{34}}+e^{\xi_{2}+\xi_{3}+\xi_{4}+A_{2}+A_{3}+A_{4}+B_{23}+B_{24}+B_{34}}, \\
g_{4}= & \xi^{\xi_{1}+\xi_{2}+\xi_{3}+\xi_{4}+A_{1}+A_{2}+A_{3}+A_{4}+B_{12}+B_{13}+B_{14}+B_{23}+B_{24}+B_{34}},
\end{aligned}
$$

where $\xi_{i}, A_{i}, g_{0}$ and $B_{i j},(i, j=1,2,3,4)$, are given by Eq. (8). By selecting the appropriate parameters

$$
\begin{aligned}
& \rho=1, \quad \omega_{1}=\frac{3}{4}, \quad \omega_{2}=\frac{1}{2}, \quad \omega_{3}=\frac{1}{3}, \quad \omega_{4}=\frac{1}{4}, \\
& \phi_{1}=1, \quad \phi_{2}=10, \quad \phi_{3}=15, \quad \phi_{4}=20, \quad \alpha_{2}=1, \quad \alpha_{3}=1,
\end{aligned}
$$

the three-dimensional and density plots of four-soliton solution $|u|^{2}$ are presented in Fig. 1.

\section{MULTI-ORDER ROGUE WAVES OF THIRD-ORDER NLSE}

In order to obtain multi-order rogue waves, we take a long wave limit with the provision each $\exp \left(\phi_{i}\right)=-1$ to Eq. (7). Then $f_{N}$ and $g_{N}$ can be written as

$$
\begin{aligned}
& f_{N}=\sum_{\mu=0,1} \prod_{i=1}^{N}(-1)^{\mu_{i}} \exp \left(\mu_{i} \eta_{i}\right) \prod_{i<j}^{(N)} \exp \left(\mu_{i} \mu_{j} B_{i j}\right), \\
& g_{N}=g_{0} \sum_{\mu=0,1} \prod_{i=1}^{N}(-1)^{\mu_{i}} \exp \left(\mu_{i} \eta_{i}\right) \prod_{i=1}^{N} \exp \left(\mu_{i} A_{i}\right) \prod_{i<j}^{(N)} \exp \left(\mu_{i} \mu_{j} B_{i j}\right),
\end{aligned}
$$


(a)

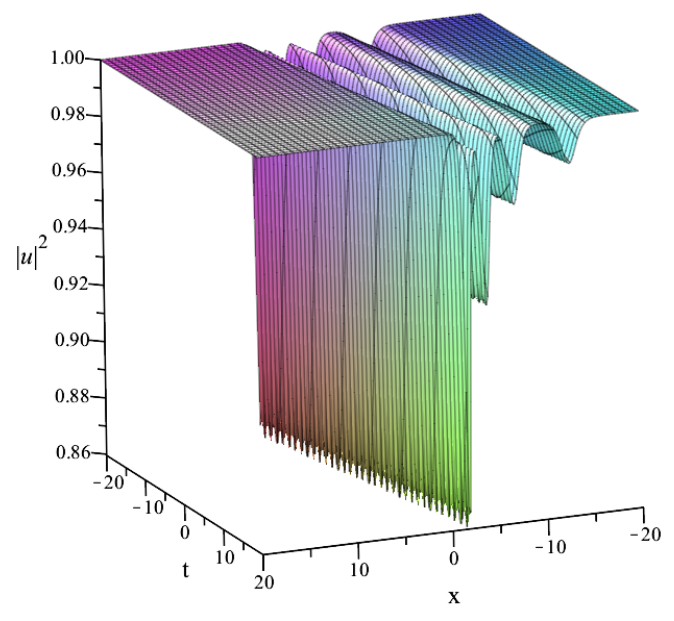

(b)

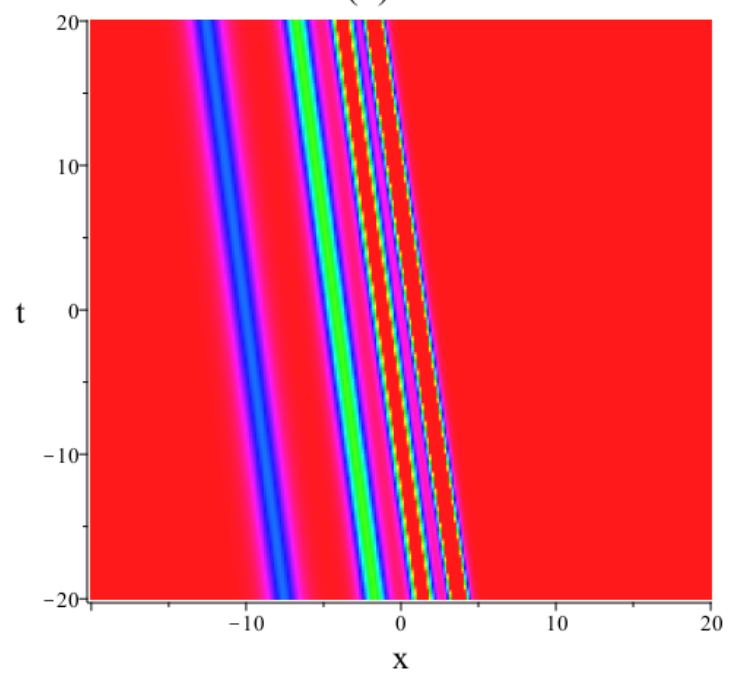

FIG. 1. (Color online) Profile of a four-soliton solution $|u|^{2}$ of Eq. (2). Fig. (a) is three-dimensional plot, (b) is density plot.

where $\eta_{i}=\delta_{i} \xi_{i}$. Taking a limit of $\delta_{i} \rightarrow 0$ and considering $\delta_{i}$ the same asymptotic order, $f_{N}$ and $g_{N}$ can be expanded as

$$
\begin{aligned}
& f_{N}=\sum_{\mu=0,1} \prod_{i=1}^{N}(-1)^{\mu_{i}}\left(1+\mu_{i} \delta_{i} \theta_{i}\right) \prod_{i<j}^{(N)}\left(1+\mu_{i} \mu_{j} \delta_{i} \delta_{j} D_{i j}\right)+O\left(\delta^{N+1}\right), \\
& g_{N}=g_{0} \sum_{\mu=0,1} \prod_{i=1}^{N}(-1)^{\mu_{i}}\left(1+\mu_{i} \delta_{i} \theta_{i}\right) \prod_{i=1}^{N}\left(1+\mu_{i} \delta_{i} C_{i}\right) \prod_{i<j}^{(N)}\left(1+\mu_{i} \mu_{j} \delta_{i} \delta_{j} D_{i j}\right)+O\left(\delta^{N+1}\right) .
\end{aligned}
$$

The leading terms of $f_{N}$ and $g_{N}$ are factorized by $\prod_{i}^{N} \delta_{i}$. Omitting the constant factor $\prod_{i}^{N} \delta_{i}$ for simplicity, the multi-soliton solutions in the long wave limit are express as

$$
\widehat{f}_{N}=\prod_{i=1}^{N} \theta_{i}+\frac{1}{2} \sum_{i, j}^{(N)} D_{i j} \prod_{l \neq i, j}^{N} \theta_{l}+\cdots+\frac{1}{M ! 2^{M}} \sum_{i, j, \ldots, m, n}^{(N)} \overbrace{D_{i j} D_{k l} \ldots D_{m n}}^{M} \prod_{p \neq i, j, \ldots, m, n}^{N} \theta_{p}+\cdots,
$$

and $\widehat{g}_{N}$ is given by changing $\theta_{k},(k=i, l, p, \ldots)$ to $\theta_{k}+C_{k}$ in $\widehat{f}_{N}$ and multiply by $g_{0}$, where

$$
\begin{aligned}
& \theta_{i}=\omega_{i} t+k_{i} x, \\
& C_{i}=-\frac{2 \alpha_{2} \omega_{i}^{2}}{i 6 \alpha_{3} \omega_{i} \rho \rho^{*}-i k_{i}} \\
& D_{i j}=\frac{2 \omega_{i}^{2} \omega_{j}^{2}\left(36 \rho \rho^{*} \alpha_{3}^{2} \omega_{i} \omega_{j}-2 \alpha_{2}^{2} \omega_{i} \omega_{j}-3 \alpha_{3} k_{i} \omega_{j}-3 \alpha_{3} k_{j} \omega_{i}\right)}{\left(k_{i} \omega_{j}-k_{j} \omega_{i}\right)^{2}} .
\end{aligned}
$$

With selecting $\theta_{M+i}=\theta_{i}^{*},(i=1,2, \ldots, M)$ for $N=2 M$, we can get a class of nonsingular rational solutions which are named as rogue wave solutions. 


\section{A. First-order rogue wave solution}

The first-order rogue wave is obtained by taking $M=1, N=2$ and $\exp \left(\phi_{i}\right)=-1$, $(i=1,2)$. By using Eq. (7), we have

$$
\begin{aligned}
& f=1-e^{\eta_{1}}-e^{\eta_{2}}+e^{\eta_{1}+\eta_{2}+B_{12}}, \\
& g=\rho e^{i 2 \rho \rho^{*} x}\left(1-e^{\eta_{1}+A_{1}}-e^{\eta_{2}+A_{2}}+e^{\eta_{1}+\eta_{2}+A_{1}+A_{2}+B_{12}}\right),
\end{aligned}
$$

where $\eta_{i}=\delta_{i}\left(\omega_{i} t+k_{i} x\right),(i=1,2)$. Taking the long wave limit method of $\delta_{i} \rightarrow 0,(i=1,2)$ and $\frac{\delta_{1}}{\delta_{2}}=O(1)$, one yield

$$
\begin{aligned}
e^{A_{i}} & =1-\frac{2 \delta_{i} \alpha_{2} \omega_{i}^{2}}{i 6 \alpha_{3} \omega_{i} \rho \rho^{*}-i k_{i}} \\
e^{B_{i j}} & =1+\frac{2 \delta_{1} \delta_{2} \omega_{i}^{2} \omega_{j}^{2} R_{i j}}{\left(k_{i} \omega_{j}-k_{j} \omega_{i}\right)^{2}}+O\left(\delta^{4}\right) \\
R_{i j} & =36 \rho \rho^{*} \alpha_{3}^{2} \omega_{i} \omega_{j}-2 \alpha_{2}^{2} \omega_{i} \omega_{j}-3 \alpha_{3} k_{i} \omega_{j}-3 \alpha_{3} k_{j} \omega_{i},
\end{aligned}
$$

and

$$
f=\delta_{1} \delta_{2}\left(\theta_{1} \theta_{2}+D_{12}+O(\delta)\right)
$$

The long wave limit of $g$ is obtained simply by substitute $\theta_{i}$ for $\theta_{i}+C_{i}$ in $f$,

$$
g=\delta_{1} \delta_{2}\left[\left(\theta_{1}+C_{1}\right)\left(\theta_{2}+C_{2}\right)+D_{12}+O(\delta)\right] \rho e^{i 2 \rho \rho^{*} x},
$$

where

$$
\begin{aligned}
\theta_{i} & =\omega_{i} t+k_{i} x \\
C_{i} & =-\frac{2 \alpha_{2} \omega_{i}^{2}}{i 6 \alpha_{3} \omega_{i} \rho \rho^{*}-i k_{i}}, \\
k_{i} & =\omega_{i}\left(6 \rho \rho^{*} \alpha_{3}-\alpha_{3} \omega_{i}^{2} \pm \sqrt{4 \rho \rho^{*} \alpha_{2}^{2}-\alpha_{2}^{2} \omega_{i}^{2}}\right), \quad i=1,2, \\
D_{12} & =\frac{2 \omega_{1}^{2} \omega_{2}^{2} R_{12}}{\left(k_{1} \omega_{2}-k_{2} \omega_{1}\right)^{2}}, \\
R_{12} & =36 \rho \rho^{*} \alpha_{3}^{2} \omega_{1} \omega_{2}-2 \alpha_{2}^{2} \omega_{1} \omega_{2}-3 \alpha_{3} k_{1} \omega_{2}-3 \alpha_{3} k_{2} \omega_{1} .
\end{aligned}
$$

In the long wave limit, the solution is yielded by using Eqs. (18) and (19)

$$
u=\rho e^{i 2 \rho \rho^{*} x} \frac{\left(\theta_{1}+C_{1}\right)\left(\theta_{2}+C_{2}\right)+D_{12}}{\theta_{1} \theta_{2}+D_{12}} .
$$

The first-order rogue wave solution is obtained by choosing $\theta_{2}=\theta_{1}^{*}$ and $D_{12}>0$. By selecting the appropriate parameters as $\rho=\frac{1}{2}, \omega_{1}=\frac{2}{3}+\frac{1}{2} i, \omega_{2}=\frac{2}{3}-\frac{1}{2} i, \alpha_{2}=\frac{1}{3}$ and $\alpha_{3}=\frac{1}{3}$, the first-order rogue wave solution is presented explicitly in Fig. 2. 

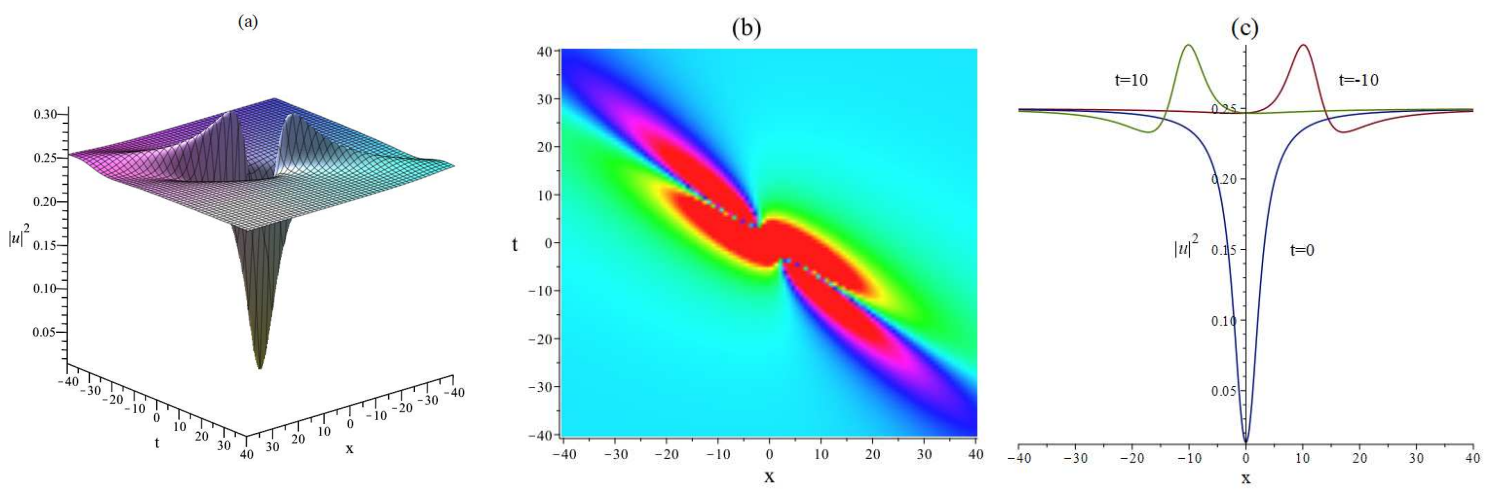

FIG. 2. (Color online) Profile of a first-order rogue wave solution of Eq. (21). (a) is threedimensional plot, (b) is density plot, (c) the wave propagation pattern along the $x$-axis by selecting different times $t=-10$ (red), $t=0$ (blue) and $t=10$ (green).

\section{B. Second-order rogue wave solution}

By taking $M=2$ and $N=4$ to Eq. (14), the second-order rogue wave $f$ and $g$ has following forms

$$
\begin{aligned}
f= & \theta_{1} \theta_{2} \theta_{3} \theta_{4}+D_{12} \theta_{3} \theta_{4}+D_{13} \theta_{2} \theta_{4}+D_{14} \theta_{2} \theta_{3}+D_{23} \theta_{1} \theta_{4} \\
& +D_{24} \theta_{1} \theta_{3}+D_{34} \theta_{1} \theta_{2}+D_{12} D_{34}+D_{13} D_{24}+D_{14} D_{24}
\end{aligned}
$$

and $g$ is given by changing $\theta_{i}$ to $\theta_{i}+C_{i},(i=1,2,3,4)$, and multiplying by $g_{0}$ of solution $f$. The second-order rogue wave solution could be obtained by choosing $\theta_{2}=\theta_{1}^{*}$ and $\theta_{4}=\theta_{3}^{*}$. The selection of parameters are $\rho=\frac{1}{2}, \omega_{1}=0.8+\frac{2}{3} i, \omega_{2}=0.8-\frac{2}{3} i, \omega_{3}=\frac{1}{2}+i, \omega_{4}=\frac{1}{2}-i$, $\alpha_{2}=\frac{1}{4}$ and $\alpha_{3}=\frac{1}{4}$. The second-order rogue wave of $|u|^{2}$ is shown in Fig. 3. By solving the relations $\left(|u|^{2}\right)_{t}=0$ and $\left(|u|^{2}\right)_{x}=0$, the positions of the maximum amplitude of rogue wave are at $(t=0.37, x=-4.85)$ and $(t=-0.37, x=4.85)$.

\section{INTERACTION BETWEEN SOLITON AND ROGUE WAVE OF THIRD-ORDER NLSE}

In order to get the interaction between one-soliton and first-order rogue wave, one take the long wave limit procedure just in $\xi_{1}$ and $\xi_{2}$. By the long wave limit provision $\exp \left(\phi_{i}\right)=-1$, $\omega_{i}=\delta_{i} \omega_{i}, k_{i}=\delta_{i} k_{i},(i=1,2), \delta_{i} \rightarrow 0$ and $N=3$, the interaction between one-soliton and 

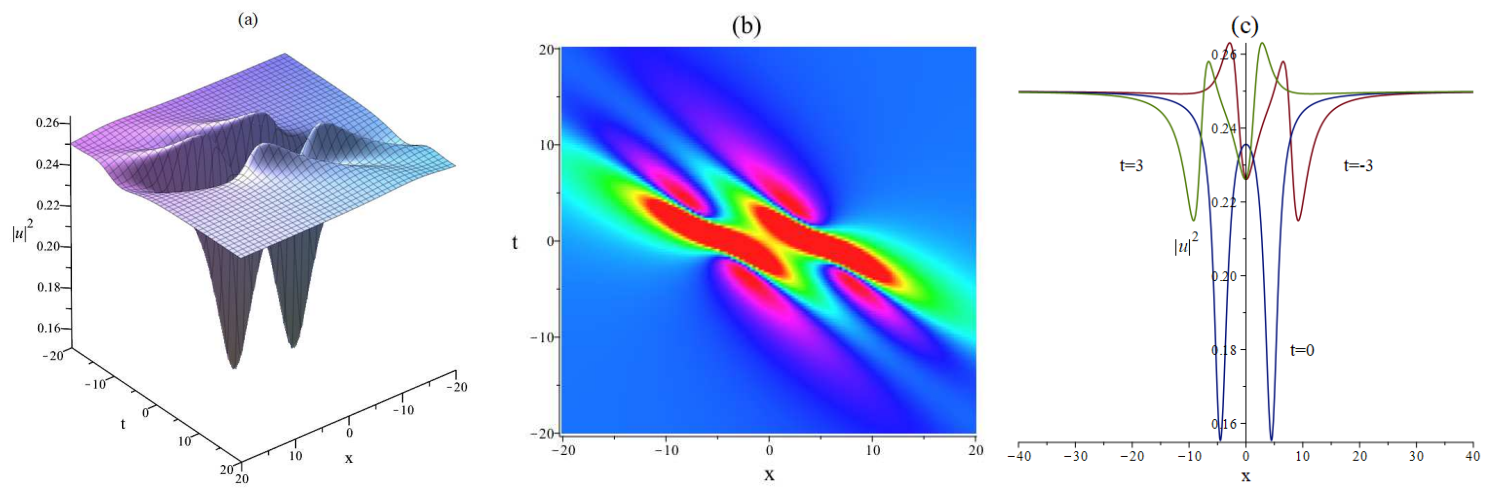

FIG. 3. (Color online) Profile of a first-order rogue wave solution $|u|^{2}$ of Eq. (2). (a) is threedimensional plot, (b) is density plot, (c) the wave propagation pattern along the $x$-axis by selecting different times $t=-3$ (red), $t=0$ (blue) and $t=3$ (green).

first-order rogue wave is obtained as

$$
\begin{aligned}
f= & \theta_{1} \theta_{2}+D_{12}+\left(\theta_{1} \theta_{2}+D_{13} \theta_{2}+D_{23} \theta_{1}+D_{12}+D_{12} D_{23}\right) e^{\xi_{3}}, \\
g= & \rho e^{i 2 \rho \rho^{*} x}\left[\theta_{1} \theta_{2}+C_{1} \theta_{2}+C_{2} \theta_{1}+C_{1} C_{2}+D_{12}+\left(\theta_{1} \theta_{2}+\left(C_{1}+D_{13}\right) \theta_{2}\right.\right. \\
& \left.\left.+\left(C_{2}+D_{23}\right) \theta_{1}+\left(C_{1}+D_{13}\right)\left(C_{2}+D_{23}\right)+D_{23}+D_{13}+D_{12}\right) e^{\xi_{3}+A_{3}}\right],
\end{aligned}
$$

where $\theta_{i}, C_{i}, D_{i j}, \xi_{3}$ and $A_{3},(i=1,2 ; j=1,2,3)$ are given by Eqs. (8) and (15). The interaction solution between one-soliton and first-order rogue wave is obtained with $\theta_{2}=\theta_{1}^{*}$. To display the solution of $|u|^{2}$, the parameters are selected as $\rho=\frac{1}{2}, \omega_{1}=\frac{4}{5}+\frac{2}{3} i, \omega_{2}=\frac{4}{5}-\frac{2}{3} i$, $\omega_{3}=\frac{1}{2}, \alpha_{2}=\frac{1}{4}$ and $\alpha_{3}=\frac{1}{4}$. The positions of rogue wave and one-soliton can be adjusted by selecting the phases $\phi_{3}$. The interaction between first-order rogue wave and one-soliton happens around $\phi_{3}=0$. By solving the solutions of $\left(|u|^{2}\right)_{t}=0$ and $\left(|u|^{2}\right)_{x}=0$, the extreme points can be obtained as $\left(t_{m}, x_{m}\right)$. The maximum amplitude of rogue wave is calculated by

$$
A=\left.\left|\lim _{x \rightarrow \infty}\right| u\left(t_{m}, x\right)\right|^{2}-\left|u\left(t_{m}, x_{m},\right)\right|^{2} \mid
$$

Fig. 4 presents the separating state of first-order rogue wave and first-order soliton with $\phi=-10$. The maximum amplitude of rogue wave is around $A=0.25$ and the corresponding extreme point is around $\left(t_{m}=0, x_{m}=0\right)$. In Fig. 5 presents overlap state of two waves. The maximum amplitude is around $A=0.11$ and the corresponding extreme point is around $\left(x_{m}=-2.02, t_{m}=0.09\right)$. The rogue wave and one-soliton will be separated with selecting lager phase $\phi_{3}$. 


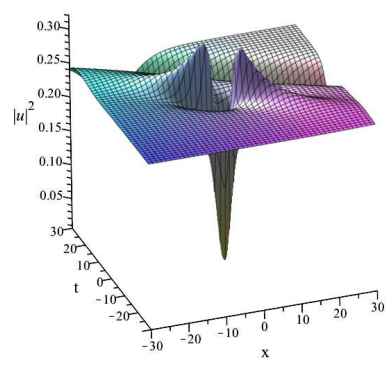

(b)

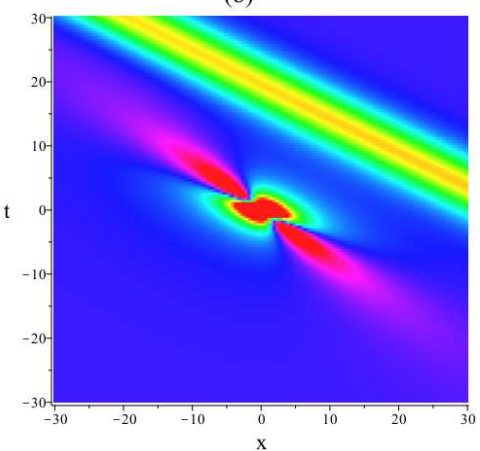

(c)

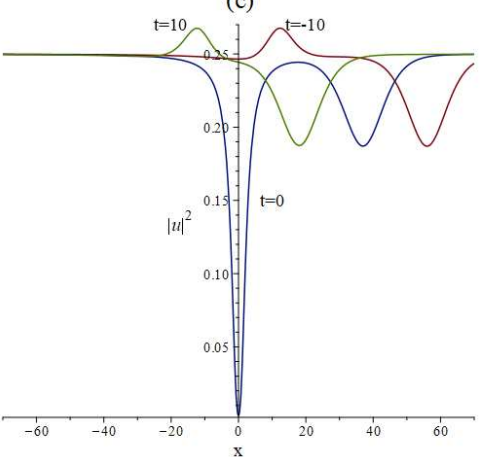

FIG. 4. (Color online) Profile of the interaction solution between first-order rogue wave and onesoliton of Eq. (2) of $\phi_{3}=-10$. (a) is three-dimensional plot, (b) is density plot, (c) the wave propagation pattern along the $x$-axis by selecting different times $t=-10$ (red), $t=0$ (blue) and $t=10$ (green).
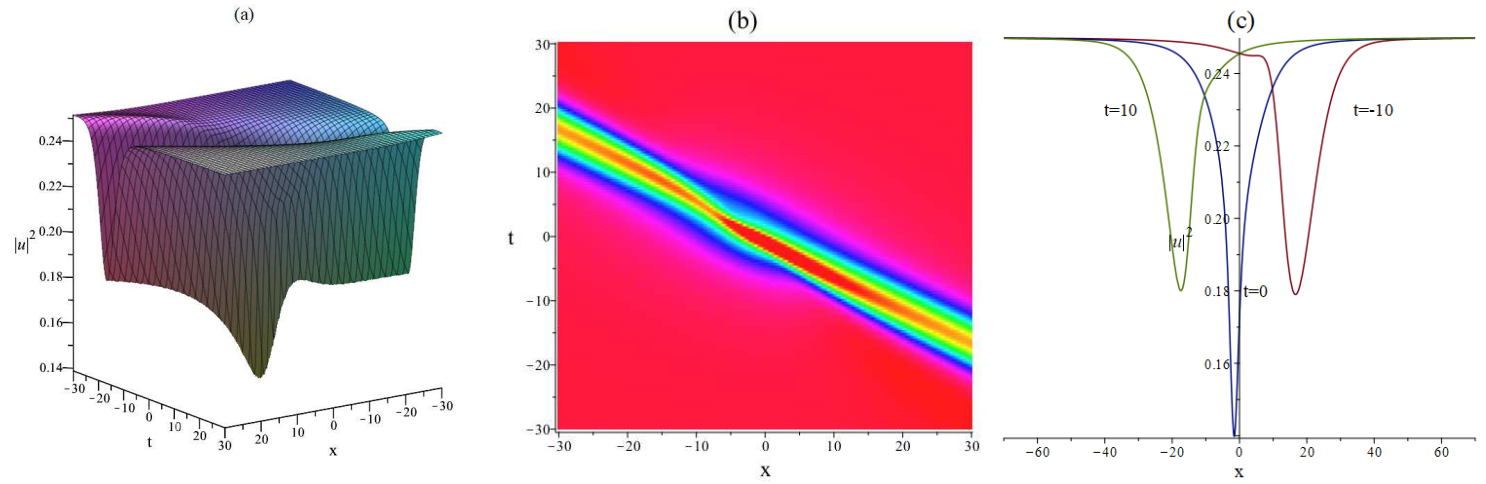

FIG. 5. (Color online) Profile of the interaction solution between first-order rogue wave and onesoliton of Eq. (2) of $\phi_{3}=0$. (a) is three-dimensional plot, (b) is density plot, (c) the wave propagation pattern along the $x$-axis by selecting different times $t=-10$ (red), $t=0$ (blue) and $t=10$ (green).

\section{CONCLUSION}

In this paper, we have investigated the multi-soliton solutions, rogue waves, interaction between rogue wave and one-soliton by the solving the bilinear form of the third-order NLSE. The dynamics of the four-soliton solution are demonstrated by taking $N=4$ in the multisolitons. Based on the long wave limit of the multi-soliton solutions, the multi-order rogue waves are derived. By taking $M=1, N=2$ and $M=2, N=4$ to the multi-order rogue 
waves, the first-order and second-order rogue waves are analyzed in details. By selecting the appropriate parameters, the dynamics of these solutions are demonstrated through by three-dimensional, density and the wave propagation pattern along the $x$-axis plots. The interaction between one-soliton and first-order rogue wave is given by applying the long wave limit procedure just in $\xi_{1}$ and $\xi_{2}$. In order to show more details of the interaction solution, the different states are demonstrated in Figs. 4 and 5. The energy of first-order rogue wave transfers to one-soliton with the phase $\phi_{3}=0$. The obtained results are expected to help the study of nonlinear optics.

\section{ACKNOWLEDGMENTS}

This work is supported by the National Natural Science Foundation of China Nos. 11775146 and 12105243 and the Natural Science Foundation of Zhejiang Province of China Grant No. LQ13A050001.

Data availability The datasets generated during and/or analyzed during the current study are available from the corresponding author on reasonable request.

\section{Declarations}

Conflict of interest The authors declare that they have no conflict of interest.

\section{REFERENCES}

${ }^{1}$ Gardner, C.S., Greene, J.M., Kruskal, M.D., Miura, R.M.: Method for solving the Korteweg-deVries equation. Phys. Rev. Lett. 19, 1095 (1967)

${ }^{2}$ Matveev, V.B., Salle, M.A.: Darboux Transformations and Solitons. Springer, Berlin (1991)

${ }^{3}$ Tang, X.Y., Lou, S.Y., Zhang, Y.: Localized excitations in (2+1)-dimensional systems. Phys. Rev. E 66, 046601 (2002)

${ }^{4}$ Debnath, L.: Nonlinear partial differential equations for scientists and engineers. Springer Science and Business Media, (2011)

${ }^{5}$ Wazwaz, A.M.: Multiple-soliton solutions for the Calogero-Bogoyavlenskii-Schiff, JimboMiwa and YTSF equations. Appl. Math. Comput. 203, 592-597 (2008) 
${ }^{6}$ Satsuma, J., Ablowitz, M.J.: Two-dimensional lumps in nonlinear dispersive systems. J. Math. Phys. 20, 1496-1503 (1979)

${ }^{7}$ Kaup, D.J.: The lump solutions and the Bäcklund transformation for the threedimensional three-wave resonant interaction. J. Math. Phys. 22, 1176-1181 (1981)

${ }^{8}$ Falcon, É., Laroche, C., Fauve, S.: Observation of depression solitary surface waves on a thin fluid layer. Phys. Rev. Lett. 89, 204501 (2002)

${ }^{9}$ Wang, X., Yang, B., Chen, Y., Yang, Y.Q.: Higher-order rogue wave solutions of the Kundu-Eckhaus equation. Phy. Scr. 89, 095210 (2014)

${ }^{10}$ Garrett, C., Gemmrich, J.: Rogue waves. Phys. Today 62, 62 (2009)

${ }^{11}$ Solli, D.R., Ropers, C., Koonath, P., Jalali, B.: Optical rogue waves. Nature 450, 1054$1057(2007)$

${ }^{12}$ Ren, B., Ma, W.X., Yu, J.: Characteristics and interactions of solitary and lump waves of a $(2+1)$-dimensional coupled nonlinear partial differential equation. Nonlinear Dyn. 96, 717-727 (2019)

${ }^{13}$ Ren, B., Cheng, X.P., Lin, J.: The (2+1)-dimensional Konopelchenko-Dubrovsky equation: nonlocal symmetries and interaction solutions. Nonlinear Dyn. 86, 1855-1862 (2016)

${ }^{14}$ Ma, W.X.: Lump solutions to the Kadomtsev-Petviashvili equation. Phys. Lett. A 379, 1975-1978 (2015)

${ }^{15}$ Ren, B., Lou, Z.M., Sun, Y.L., He, Z.W.: Dynamical analysis of solitary waves, lumps and interaction phenomena of a (2+1)-dimensional high-order nonlinear evolution equation. Mod. Phy. Lett. B 33, 1950181 (2019)

${ }^{16}$ Ren, B., Ma, W.X., Yu, J.: Rational solutions and their interaction solutions of the $(2+1)-$ dimensional modified dispersive water wave equation. Comput. Math. Appl. 77, 2086-2095 (2019)

${ }^{17}$ Ren, B., Lin, J., Lou, Z.M.: A new nonlinear equation with lump-soliton, lump-periodic, and lump-periodic-soliton solutions. Complexity 4072754, 2019 (2019)

${ }^{18}$ Rao, J.G., Zhang, Y.S., Fokas, A.S., He, J.: Rogue waves of the nonlocal Davey-Stewartson I equation. Nonlinearity 31, 4090 (2018)

${ }^{19}$ Zhang, Y., Liu, Y.P., Tang, X.Y.: M-lump solutions to a (3+1)-dimensional nonlinear evolution equation. Comput. Math. Appl. 76, 592-601 (2018)

${ }^{20}$ Qian, C., Rao, J.G., Liu, Y.B., He, J.S.: Rogue Waves in the Three-Dimensional Kadomtsev-Petviashvili Equation. Chin. Phys. Lett. 33, 110201 (2016) 
${ }^{21}$ Wang, C.J., Nie, Z.Z., Xie, W.J., Gao, J.Y., Zhou, Q., Liu, W.J.: Dark soliton control based on dispersion and nonlinearity for third-order nonlinear Schrödinger equation. Optik, 184, 370-376 (2019)

${ }^{22}$ Rao, J.G., Liu, Y.B., Qian, C., He, J.S.: Rogue waves and hybrid solutions of the Boussinesq equation. Z. Naturforsch. 72a, 307-314 (2017).

${ }^{23}$ Satsuma, J., Ablowitz, M.J.: Two-dimensional lumps in nonlinear dispersive systems. J. Math. Phys. 20, 1496-1503 (1979)

${ }^{24}$ Imai, K.: Dromion and lump solutions of the Ishimori-I equation. Prog. Theor. Phys. 98, $1013(1997)$

${ }^{25}$ Estévez, P.G., Prada, J., Villarroel, J.: On an algorithmic construction of lump solutions in a $2+1$ integrable equation. J. Phys. A: Math. Theor. 40, 7213-7231 (2007)

${ }^{26}$ Dai, Z.D., Liu, Z.J., Li, D.L.: Exact periodic solitary-wave solution for KdV equation. Chin. Phys. Lett. 25, 1531 (2008)

${ }^{27}$ Jiang, Y., Tian, B., Li, M., Wang, P.: Bright hump solitons for the higher-order nonlinear Schrödinger equation in optical fibers. Nonlinear Dyn. 74, 1053-1063 (2013)

${ }^{28}$ Ma, L.Y., Zhang, Y.L., Tang, L., Shen, S.F.: New rational and breather solutions of a higher-order integrable nonlinear Schrödinger equation. Appl. Math. Lett. 122, 107539 (2021)

${ }^{29}$ Xiang, X.S., Zuo, D.W.: Breather and rogue wave solutions of coupled derivative nonlinear Schrödinger equations. Nonlinear Dyn. 164 1-10 (2021)

${ }^{30}$ Fibich, G.: The nonlinear Schrödinger equation. Springer, Berlin (2015)

${ }^{31}$ Agrawal, G.P.: Nonlinear Fiber Optics. Academic Press, San Diego (1995)

${ }^{32}$ Wang, L., Zhang, J.H., Wang, Z.Q., et al.: Breather-to-soliton transitions, nonlinear wave interactions and modulational instability in a higher-order generalized nonlinear Schrödinger equation. Phys Rev E. 93012214 (2016) 